\title{
Performance Evaluation of Heterogeneous Sensor Networks with ATPC and Load Balancing
}

\author{
Sudarmani.R \\ Assistant Professor/ECE department \\ Faculty of Engineering \\ Avinashilingam Deemed University \\ for Women, Coimbatore
}

\author{
Dr.K.R. Shankar Kumar \\ Professor/ECE department \\ Sri Ramakrishna Engineering College \\ Coimbatore
}

\begin{abstract}
Wireless Sensor Networks (WSNs) are battery-powered ad-hoc networks in which sensor nodes are scattered over a region. These networks have high node density but highly resourceconstraint in terms of size, power and bandwidth. Most of the existing sensor networks focus on homogeneous sensor networks, but these networks have poor performance in terms of energy consumption and network life time. So it is required to analyse the heterogeneous sensor network (HSN) model for better performance. Adjusting the transmission power of each node instead of using same power level to all nodes is the method used in HSN to reduce energy consumption. HSN with adaptive transmission power control (ATPC) of low-end sensors are analysed in terms of energy consumption and network life time and compared it with HSN without power control. But ununiform load distribution has been taken place. In this paper, Load balancing is also applied over HSN with ATPC and it is compared with HSN with ATPC. Simulation results prove that heterogeneous sensor networks with ATPC and Load balancing outperform the heterogeneous sensor networks with ATPC alone in terms of energy consumption and network life time.
\end{abstract}

\section{Keywords}

Heterogeneous sensor networks, sensor nodes, clustering, transmission power control, load balancing.

\section{INTRODUCTION}

Advances in wireless networking, micro-fabrication and integration (for example, sensors and actuators manufactured using micro-electromechanical system (MEMS) technology), and embedded microprocessors have enabled a new generation of massive-scale sensor networks suitable for a range of commercial and military applications. The technology promises to revolutionize the way we live, work, and interact with the physical environment. In a typical sensor network, each sensor node operates untethered and has a microprocessor and a small amount of memory for signal processing and task scheduling [4]. Each node is equipped with one or more sensing devices such as acoustic microphone arrays, video or still cameras, infrared (IR), seismic, or magnetic sensors.

Each sensor node communicates wirelessly with a few other local nodes within its radio communication range.But currently existing wireless sensor networks consist of homogeneous types of sensors, which comprise identical sensors with equal capacity in terms of sensing, computation, communication and power. Hence they become application specific. In this paper, heterogeneous sensor network is utilised, which consists of different compositions of sensors, for example some sensors collect image data, some collect audio signal, some have more processing capabilities, some have more power etc. Thus, various operations are performed simultaneously.

Collecting the sensed data from sensor nodes in a wireless sensor network occurs in different ways. The simplest is direct transmission, where each sensor directly sends gathered information to the remote receiver independent of each other.

This approach has an inherent scalability problem. A second approach is via multi-hop routing, which has been extensively used for both generic ad-hoc routing networks as well as wireless sensor networks. These multi-hop routing techniques would perform the communications for small number of receivers. The third method is clustering where sensors form clusters dynamically with neighbouring sensors. One of the sensors in the cluster will be elected as cluster head. The elected cluster head will be responsible for relaying data from each sensor in the cluster to the remote receiver. In addition, data fusion and data compression can occur in the cluster head by considering the potential correlation among data from neighbouring sensors. This clustering approach is preferred widely because it localizes traffic and can potentially be more scalable $[1,2,3,5]$. To increase network life time, the energy consumption for communication must be reduced for HSN. It is done by reducing transmission power level of sensor nodes. At the same time load balancing with in the clusters has to be taken place to increase the network life time of HSN. 
In this paper, performance parameters of clustered heterogeneous sensor networks with load balancing and adaptive transmission power control over sensors are analysed and compared with HSN with ATPC and with out load balancing. Power control can be taken as a network layer problem [8]. The paper is organized as follows. In section II, heterogeneous sensor network model is analyzed, section III is about the proposed system, section IV deals with the protocols used in our system, section $\mathrm{V}$ about the energy consumption analysis of our system. Finally, concluded with simulation and results.

\section{HETEROGENEOUS SENSOR NETWORK MODEL}

Heterogeneous sensor network model consists of two physically different types of sensor nodes: powerful HighEnergy sensors (HE) and Low-Energy sensors (LE) [4]. Both LE-sensors and HE-sensors are uniformly and randomly distributed in the field. After sensor deployment, clusters are formed in HSN. The powerful HE-sensors serve as cluster heads and form clusters around them. All the HE-sensors form a backbone in the network. The cluster formation is shown in Fig.1, it consists of LEsensors, HE-sensors and the Base station (BS).

Powerful HE-sensors have more energy supply, longer transmission range, higher data rate than LE-sensors and they provide many advantages for designing better protocols, algorithms, and secure schemes in sensor networks. An efficient and robust cluster formation scheme with well organized routing protocol is adopted for HSN. Usually, each sensor node has a sensing radius and it is required that the sensor nodes provide coverage of the region with a high probability [8]. The sensing radius of each node depends on the phenomenon that is being sensed as well as the sensing hardware of the node. During each data gathering cycle, the sensor nodes send their sensed data to the closest cluster head node which performs data aggregation. Then the cluster head directly transmits the aggregated data to a base station (assumed to be remotely located).

The sensor nodes have simple functionality, since they perform sensing and relatively short range communication. However the cluster head nodes are more complex, since they co-ordinate MAC and routing within their cluster, perform data fusion, and perform long range transmissions to the remote base station.

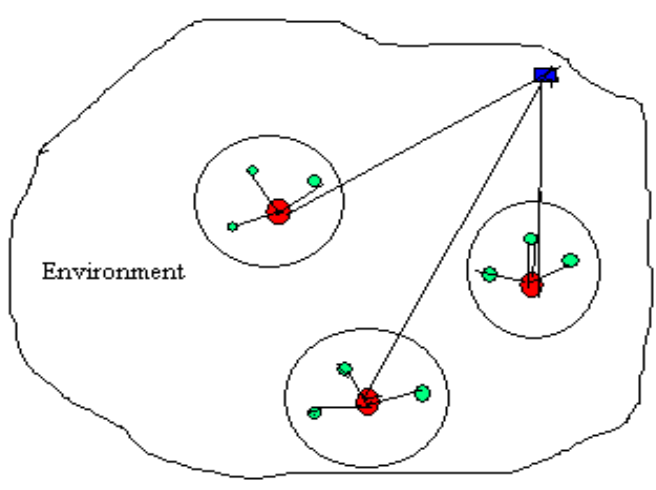

High energy sensor
Low energy sensor
$\square$ Sink

Fig.1 Hierarchical network model

\section{HETEROGENEOUS SENSOR NETWORK WITH ATPC}

In a hierarchical sensor network, the nodes are randomly or deterministically distributed over a given area. The HE nodes transmit hello packets to all the LE nodes and LE nodes in turn acknowledge the receipt of the signal. Sensors are organized within clusters based on the shortest distance between $\mathrm{HE}$ and LE nodes. After grouped as clusters, the LE nodes reduce its transmission power level according to the distance of the corresponding HE nodes.

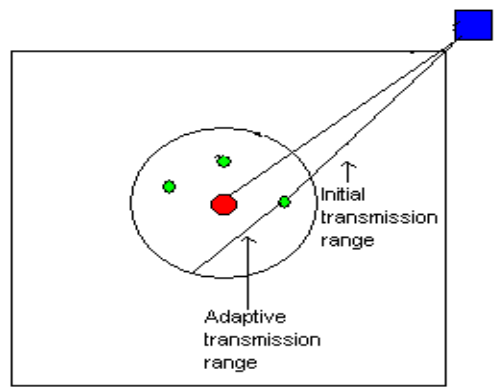

Fig.2 Hierarchical network with adaptive transmission range

All HE nodes have to gather the sensed data within the cluster and to forward it to a unique base station denoted as sink. Two main problems can be addressed for this general architecture: the inter- $\mathrm{CH}$ routing and the intra cluster data gathering. Direct transmission is used between CHs and sink. The Cluster Heads have larger energy and processing capacities than the LE sensors. In fact, sensing nodes just have to sense information and send it to the corresponding HE-nodes whereas the HE nodes have to 
manage their clusters, do data processing and delivering and relay data for inter cluster communication.

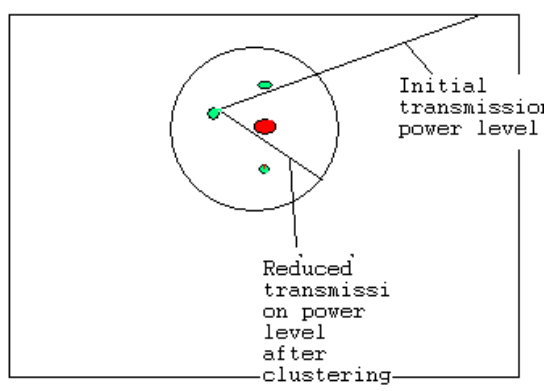

Fig.3 Hierarchical network with ATPC

Each Cluster Head is responsible of organizing communications inside its cluster. The initial energy of the cluster head should be optimally shared between the two $\mathrm{CH}$ activities: intra-cluster management (scheduling, aggregation ...) and data relaying (inter cluster routing). The energy optimization and network life time are simultaneously done by adjusting the transmission power level of each LE node, the adaptive transmission power level has taken from [9]. At the same time transmission range also reduced for LE nodes. Round, the term involved here is the period in which the sensed data is transferred from $\mathrm{LE}$ to receiver through $\mathrm{HE}$ nodes. If the $\mathrm{HE}$ sensor decides not to be the cluster head, then it goes to the sleep mode. Round ends when the data from all the sensors are relayed to the collector.

\section{PROPOSED SCHEME}

The sensor network is denoted by undirected graph $\mathrm{G}(\mathrm{V}$, $\mathrm{E}$ ), where $\mathrm{V}$ denotes the set of all nodes which includes $\mathrm{HE}$ nodes and LE nodes. In that $10 \%$ of nodes are HE nodes.

$E$ denotes the set of links between any pair of nodes in the radio range $\mathrm{R}$. The initial energy of $\mathrm{LE}$ and $\mathrm{HE}$ sensors are represented by $\mathrm{E}_{\mathrm{LEi}}$ and $\mathrm{E}_{\mathrm{HEi}}$ respectively. The $\mathrm{HE}$ sensors are having high energy, that is $\mathrm{E}_{\mathrm{HEi}}>\mathrm{E}_{\mathrm{LEi}}$. The algorithm consist of two phases, that are Cluster formation phase with Load balancing and Adaptive transmission power control phase.

(a). Cluster formation phase with Load balancing

HE nodes and LE nodes are uniformly and randomly distributed in the network area. All HE nodes flood the Hello packets, after some delay in turn receives the ACK message. Initially the LE nodes transmission range covers the entire network, after receiving the ACK from LE nodes, each HE node calculates distance between LE nodes and itself, it is represented by $\mathrm{D}_{\mathrm{LH}}$. Let $\mathrm{D}_{\text {th }}$ be the threshold distance. After calculating the distance $\mathrm{D}_{\mathrm{LH}}$ by all $\mathrm{HE}$ nodes, it is compared with $\mathrm{D}_{\text {th }}$ and the nodes which are less than the threshold distance are connected to the particular $\mathrm{HE}$ node till the counter associated with the HE nodes become zero to obtain load balancing.

(b). Adaptive transmission power control phase

Let $\mathrm{R}$ be the initial transmission range of all nodes. After grouping as clusters the transmission power level of each LE nodes with in the clusters are adjusted according to the distance between HE and LE nodes.

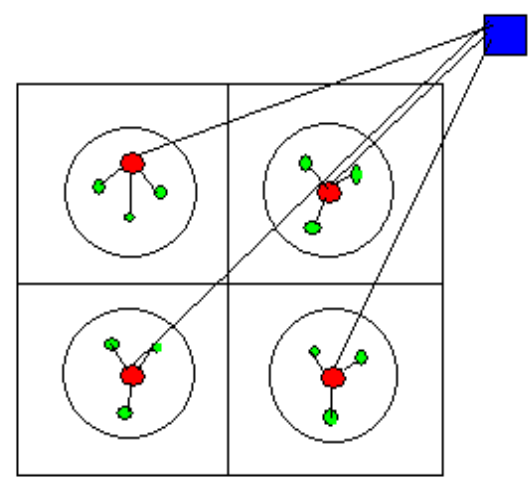

Fig.4 Hierarchical network with Load Balancing and ATPC

\section{PROTOCOLS USED}

Media Access Control (MAC) protocol version IEEE 802.11 is used to avoid energy wastage due to collision, overhearing, control packet overhead, idle listening, over emitting etc. The reasons behind adopting this protocol are, it is energy efficient, scalable and the nodes can be taken to sleep mode once they are inactive thereby avoiding energy wastage.

AODV-Ad-hoc on demand Distance Vector is the routing protocol used in dynamic wireless network where nodes can enter and leave the network at will. In this protocol the source node sends route request to the neighbouring nodes, if there exists a path from the node to destination, the node acknowledges to the source node. If not, the node forwards back the same request to the source.

Communication protocols used are User Datagram Protocol (UDP) and Transmission Control Protocol (TCP). In UDP, bit rate is constant and packet size can be varied while communicating and in TCP , every communication is acknowledged and thus, the communication becomes reliable.

\section{ENERGY CONSUMPTION ANALYSIS IN HSN WITH ATPC}

The number of sensor nodes required in a region to be sensed is determined by the application requirements. The 
network is heterogeneous and there are two types of nodes; cluster head nodes (HE) and sensor nodes (LE). The cluster head nodes act as the fusion points within the network. During each data gathering cycle, the sensor nodes send their sensed data to the closest cluster head node which performs data aggregation. Then the cluster head directly transmits the aggregated data to a base station (assumed to be remotely located). The LE nodes have simple functionality, since they perform sensing and relatively short range communication. However the cluster head nodes (HE) are more complex, since they co-ordinate MAC and routing within their cluster, perform data fusion, and perform long range transmissions to the remote base station(more battery energy). The overall system design problem involves determining the optimum number of cluster head nodes. The cluster is a circular region and the cluster head is located at the center of this region. Let the communication between LE nodes with their HE node will be single hop and communication between HE node and base station is also single hop. Assume a propagation loss constant of $\mathrm{k}$ for communication within a cluster, and $\mathrm{k}_{0}$ for communication between the HE nodes base station. Since the cluster head to base station communication is long range, it is likely that $\mathrm{k}_{0}>\mathrm{k}$. The exact values of $\mathrm{k}$ and $\mathrm{k}_{0}$ depend on the environment in which the network operates. When the sensor nodes use single hop communication,

there is no relaying of packets. Each node directly transmits its packet to the cluster head (see Fig. 1). The communication area of each node is smaller than the total area of the cluster.

The total energy consumption of heterogeneous sensor networks is obtained by combining the energy consumed by cluster heads and non cluster heads. The total energy consumed by heterogeneous sensor networks [10] is given by

$$
E_{T}=E_{H E}+E_{L E}-(1)
$$

Where,

$$
\begin{array}{r}
E_{H E}=T\left(\frac{n_{0}}{n_{1}} l_{1}+E_{f}+l_{2}+\mu_{2} d^{4}\right)-(2) \\
E_{L E}=T\left(l_{1}+\frac{\mu_{1} A^{2}}{n_{1}}\right)-(3)
\end{array}
$$

$E_{f}$ is the computational energy spent on fusion of each packet $l_{1} \quad$ is the amount of energy spent in the transmitter electronics circuitry within a cluster

$l_{2}$ is the amount of energy spent in the transmitter electronics circuitry from the cluster head to the base station

$\mu_{1}$ is the energy spent in the RF amplifier within the cluster

$\mu_{2}$ is the energy spent in the RF amplifier from the cluster head to the base station

$\mathrm{A}$ is the radius of the region

$\mathrm{T}$ is the data gathering cycles

$n_{0}$ is the number of low energy nodes

$n_{1}$ is the number of high energy nodes

$\frac{A}{\sqrt{n_{1}}}$ is the radius of the cluster region

In this paper, the power control functionality is introduced based on the distance between LE and HE nodes. According to the distance between them, the LE nodes reduce its transmitter power adaptively. Hence, reduction in the power consumption takes place according to equation (1) at the same time network life increases compared with HSN.

\section{SIMULATION RESULTS}

\subsection{Simulation Environment}

Network simulator ns-2 is used for simulation. Two ray ground reflection model is used and nodes are spread in a square region, having dimension of $200 \mathrm{~m}$ x $200 \mathrm{~m} .100$ nodes are uniformly placed over the area. Out of 100 nodes $10 \%$ of nodes are HE nodes. One base station is located faraway from the nodes.

\subsection{Simulation Results}

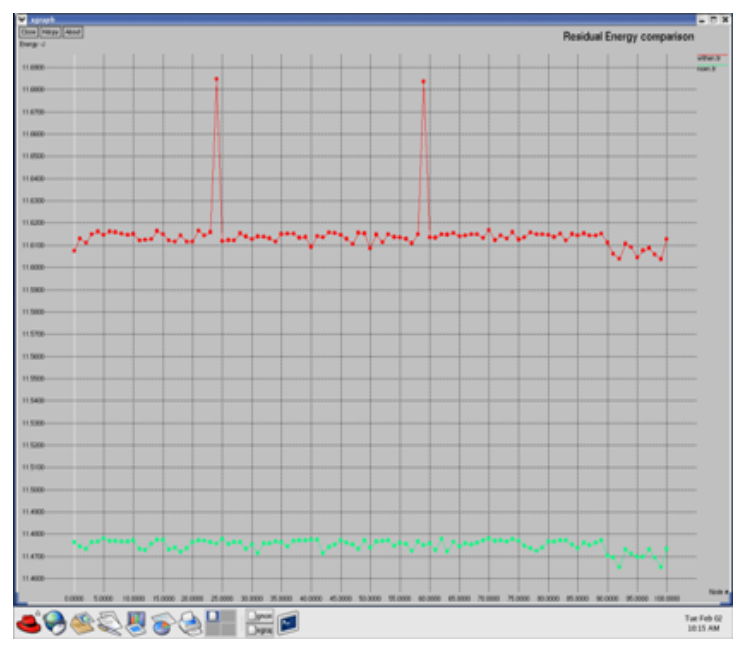

Fig.5. Residual energy comparison graph 
For one packet transmission the HSN with transmission power control can save up to 4 joules of energy for 100 nodes when compared to HSN as in fig. 5.

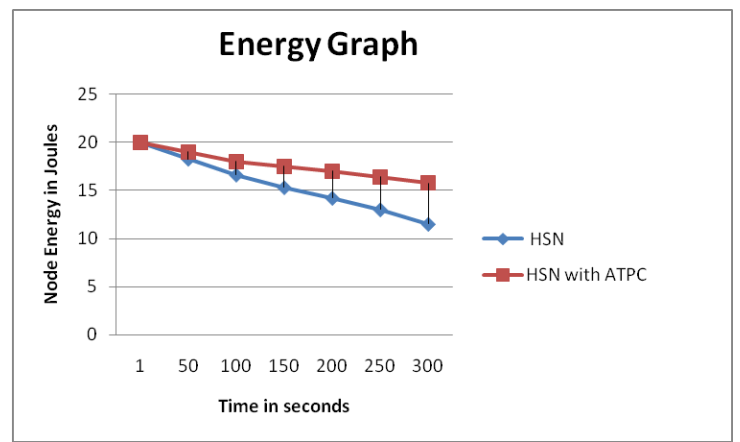

Fig. 6 Comparison graph of Energy depletion rate

Fig. 6 shows that after the 300 second simulation time the residual energy of HSN with ATPC is more compared to the HSN. Hence the life time of HSN with ATPC is longer compared to HSN.

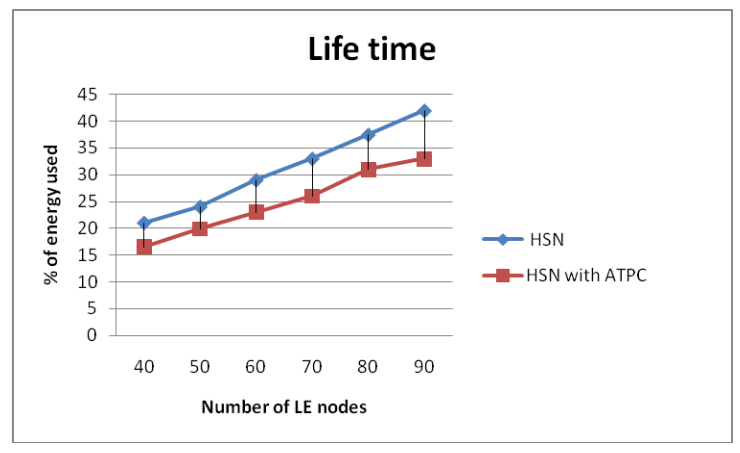

Fig.7 Network Life time

Simulation is carried out for calculating energy consumption by varying the number of LE nodes. The plot of the number of LE nodes Vs energy consumption is shown in fig. 7. It shows that the energy consumed by HSN with ATPC is less when compared to HSN. Hence optimization of life time as well as energy consumption is achieved in the case of HSN with ATPC.

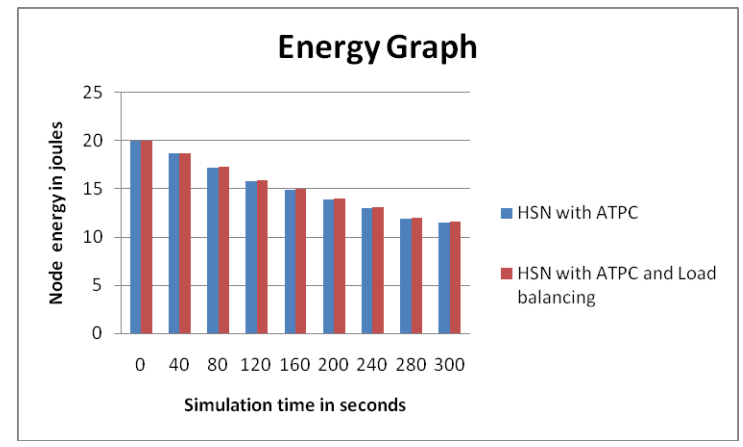

Fig. 8 Residual Energy comparison graph of HSN with ATPC and load balancing and HSN with ATPC.

Application of Load balancing leads to more residual energy than HSN with ATPC. When more packetes are transmitted the energy is saved, hence life time is also increased.

\section{CONCLUSION}

Heterogeneous sensor network with ATPC is implemented and compared with heterogeneous sensor network for energy consumption and network life time. HE sensors have longer transmission range, hence number of hops to reach receiver is reduced at the same time LE sensors reduces its transmission range and thus energy optimization is obtained. Load balancing is applied to HSN with ATP and compared with HSN with ATPC. From the simulation results the energy optimization and network lifetime obtained by the proposed method are better compared to the HSN with ATPC.

\section{REFERENCES}

[1] S. Bandyopadhyay and E. J. Coyle, "Minimizing Communication Costs in Hierarchically Clustered Networks of Wireless Sensors", WCNC 2003.

[2] G. Gupta and M. Younis, "Load-Balanced Clustering in Wireless Sensor Networks", ICC 2003.

[3] W. B. Heinzelman, A. P. Chandrakasan, and H. Balakrishnan, "An Application-Specific Protocol Architecture for Wireless Microsensor Networks", IEEE Transactions on Wireless Communications, Vol. 1, No. 4, pp.660-670, Oct. 2002.

[4] Iaojiang Du, Fengjing Lin, "Improving Routing in Sensor Networks with Heterogeneous Sensor Nodes” Proceedings of IEEE Vehicular Technology Conference(VCT'05), Stockholm, Sweden, vol.4, pp.2528-2532, June 2005.

[5] E-S. Jung and N. H. Vaidya, "A Power Control MAC Protocol for Ad Hoc Networks", MOBICOM 2002.

[6] H. Karl and A. Wilig, Protocols and Architectures for Wireless Sensor Networks, John Wiley \& Sons, 2005. 
[7] V. Kawadia and P. R. Kumar, "Principles and Protocols for Power Control in Wireless Ad Hoc Networks", WEEE Journal on Selected Areas in Communications, Vol. 23, No. 12, pp. 76-88, Jan. 2005.

[8] J. S. Liu and C. H. R. Lin, "Energy-Efficiency Clustering Protocol in Wireless Sensor Networks", Ad Hoc Networks, Vol. 3, pp. 371-388, 2005.

[9] P. Santi, Topology Control in Wireless Ad Hoc and Sensor Networks, John Wiley \& Sons, 2005.
[10] R.Sudarmani, K.R. Shankar Kumar, “Adaptive Transmission Power Control based Energy Consumption Analysis in Heterogeneous Sensor Networks", Journal of Computing, Vol. 2, issue 8, Aug. 2010.

[11] D. Wei and H. A. Chan, "A Survey on Cluster Schemes in Ad Hoc Wireless Networks", 2nd International Conference on Mobile Technology, Applications and Systems, pp. 1-8,Nov. 2005. 